\title{
Fantoms
}

Ben Stenson, Deputy Editor

\section{Predicting outcome}

Cerebral ultrasound and, increasingly, MRI scan findings are the focus of so many difficult conversations between families and the healthcare team regarding prediction of the future. The difference between population estimates of risk and the real life outcomes of individual babies is so hard to encapsulate. Nongena et al provide a very helpful summary of published data linking neuroimaging findings in preterm infants to later outcome, broken down into readily separable categories for each imaging modality. Their concluding sentence is an excellent starting point for anyone. "Wise clinicians have always known that individual tests rarely provide certainty, and they continue to use neuroimaging with circumspection." See page 388

The 2005 International Liaison Committee on Resuscitation (ILCOR) consensus on science and treatment recommendations for neonatal resuscitation say that "If there are no signs of life after 10 minutes of continuous and adequate resuscitative efforts, it may be justifiable to stop resuscitation." This was based on a literature that predicted a poor outcome in virtually all cases. The data pre-dated the introduction of therapeutic hypothermia. Earlier this year Laptook et al ${ }^{1}$ published outcome data for 25 infants with an Apgar score of 0 at $10 \mathrm{~min}$ who were enrolled in a hypothermia trial which suggested that adverse outcome may no longer be quite as confidently predicted. In this issue, Sarkar et al examine precooling predictors of adverse outcome from hypoxic ischaemic encephalopathy in 85 infants who were treated with hypothermia. Twelve of the infants had Apgar scores of 0 at 5 and $10 \mathrm{~min}$. Nine of the 12 died and the 3 survivors had evidence of extensive brain injury. Eight of the nine deaths occurred after completion of hypothermia treatment. It is difficult to know the true denominator for case series like these because many infants with an Apgar score of zero at 10 min may not get as far as commencing hypothermia. Further data from larger populations are required - a good reason to ensure that all children who are treated with hypothermia are registered. A revised ILCOR statement is due in November 2010. See page 423 .

\section{Transport}

Continuing a theme of hypothermia, Kendall et al present the temperature profiles of 39 infants who were transferred for therapeutic hypothermia and had cooling provided during transport. Infants were cooled using passive measures (allowing the infant to cool naturally with no external intervention) following a protocol from the TOBY group (https:// www.npeu.ox.ac.uk/tobyregister/ transport) and $67 \%$ of them were within the target temperature range on arrival at their destination (compared to $15 \%$ at the start of the transport episode). Overand undercooling occurred. The authors suggest that rectal temperature monitoring is essential for any infant undergoing passive or active cooling - skin or axillary temperature being unreliable. Initiation of therapeutic hypothermia is time-critical, and several UK neonatal transport services have now adapted servo-controlled systems for use in transport. See page 408

Mohamed and Aly analyse data from 67000 very low birth weight infants born between 1997 and 2004. 9.2\% of them were transferred between hospitals in the first $48 \mathrm{~h}$ after birth and, after controlling for other factors, transferred infants remained significantly more likely to develop intraventricular haemorrhage (OR 1.75 (1.64-1.86), $\mathrm{p}<0.001$ ). This is consistent with UK data from the first EPICURE study and is a reason for ensuring that wherever possible these infants are delivered in a hospital that is capable of providing the ongoing care rather than transported after birth. Whether it is the transport itself that is responsible for the association or some aspect of the pretransport events is less clear. See page 403

\section{Initial stabilisation}

Schmolzer et al provide detailed information regarding tidal volume, airway pressures and mask leaks in newborn infants receiving positive pressure ventilation immediately after birth. They combine this with clinical observations and show the difficulty of making clinical assessments in these situations. There is lots of fascinating information in this paper, not least the wide range of tidal volumes administered to preterm infants during their initial stabilisation after birth. This might in part be due to the difficulty that so many people have in mask ventilating these small babies without intermittently obstructing the airway. Airway obstruction during mask ventilation is the subject of another paper from the same group, this time evaluating the ventilation of a manikin. See pages 393 and 398

\section{Sucrose analgesia}

O'Sullivan et al studied the effect of sucrose analgesia for retinopathy of prematurity screening examinations. Sucrose was found to lower pain scores when compared with placebo but the scores remained high enough to suggest that the infants were still experiencing significant discomfort that was not being alleviated. See page 419

\section{Glucose homeostasis in preterm infants}

Chacko and Sunehag provide fascinating data regarding glucose homeostasis in preterm infants. Stable, well infants (mean, range blood glucose 8.9, range $5.2-14.3 \mathrm{mmol} / \mathrm{l}$ ) continued to produce glucose by both gluconeogenesis and glycogenolysis even when their glucose infusion rates from total parenteral nutrition averaged $10 \mathrm{mg} / \mathrm{kg} / \mathrm{min}$. This glucose production was not related to blood glucose level, or infusion rates of glucose, lipid or amino acids. See page 413

\section{REFERENCE}

1. Laptook AR, Shankaran S, Ambalavanan N, et al.; Hypothermia Subcommittee of the NICHD Neonatal Research Network. Outcome of term infants using apgar scores at 10 minutes following hypoxic-ischemic encephalopathy. Pediatrics 2009:124:1619-26. 